\title{
Preparation and characterization of a stereocomplex of poly(lactide-co-e-caprolactone)/tricalcium phosphate biocomposite using supercritical fluid technology
}

\author{
R. I. Nurqadar ${ }^{1,2}$, P. Purnama ${ }^{1}$, S. H. Kim ${ }^{1,2,3^{*}}$ \\ ${ }^{1}$ Biomaterials Research Center, Korea Institute of Science and Technology, 136-791 Seoul, Korea \\ ${ }^{2}$ Biomedical Engineering, University of Science and Technology, 113 Gwahangno, Yuseong-gu, 305-333 Daejeon, Korea \\ ${ }^{3}$ KU-KIST Graduate School of Converging Science and Technology, Korea University, 136-701 Seoul, Korea
}

Received 30 May 2013; accepted in revised form 13 August 2013

\begin{abstract}
A novel biocomposite material from a stereocomplex of poly (L-lactide-co-e-caprolactone) (PLLCL) and poly (D-lactide-co- $\varepsilon$-caprolactone) (PDLCL) and inorganic tricalcium phosphate (TCP) was prepared by supercritical fluid method. Both pristine and poly (L-lactide)-grafted-TCP (PLLA-g-TCP) were used. PLLA-g-TCP was produced by ringopening polymerization of L-lactide in the presence of surface-activated TCP. Infrared (IR) spectroscopy and scanning electron microscopic (SEM) images confirm the attachment of PLLA onto the activated TCP surface. The stereocomplex formation of biocomposite was confirmed by differential scanning calorimetry (DSC) and wide-angle X-ray diffraction (WAXD). The biocomposite containing PLLA-g-TCP has higher stereocomplex degree and more homogeneous TCP distribution compared to the biocomposite containing pristine TCP. The presence of PLLA-g-TCP in the stereocomplex PLLCLPDLCL (s-PDLCL) enhance the stereocomplex degree up to a certain content and also supports the homogeneous TCP dispersion in the stereocomplex matrix. These phenomena support the improvement in mechanical properties of the s-PDLCL composite the optimum content of PLLA-g-TCP being 10\%. The biocomposites containing TCP materials are promising materials for biomedical application, especially for bone tissue engineering.
\end{abstract}

Keywords: biocomposites, polylactide, biodegradable polymers, stereocomplex

\section{Introduction}

Absorbable materials are attractive for internal fracture fixation devices. They have advantages in the reduction of stress shielding, secondary operations for removal are no longer required, and the risks of complications are reduced [1]. Biopolymers such as polylactide, poly(glycolic acid), poly(e-caprolactone), and their copolymers attract great attention due to their biodegradability and biocompatibility. The major limitation of those materials is that their mechanical properties (as strength, toughness, elastic modulus) are lower than the mechanical properties of cortical bones.
Polylactide-based materials have attracted great interest due to their capability to form stereocomplex. Stereocomplex can be formed by interaction between L-lactide and D-lactide fragments with minimum length of about 7 lactide units [2]. The stereocomplexation caused by interaction of $\mathrm{L}-$ and D-lactide sequences affect the mechanical and thermal properties of polylactide [3]. The linear stereocomplex polylactide has limitation in melt stability. This drawback can be reduced by adding flexible fragments such as $\varepsilon$-caprolactone to the polylactide [4]. Improvement in mechanical and thermal performance of polylactide by stereocomplexation still

\footnotetext{
${ }^{*}$ Corresponding author, e-mail: soohkim@kist.re.kr (C) BME-PT
} 
does not match with cortical bone level. For this reason, there are many developments to prepare absorbable material with proper mechanical properties to match natural bones by combining biodegradable polymers and inorganic fillers.

There are many inorganic fillers capable of improving the mechanical performance and bioactivity of composites such as hydroxyapatite, TCP, bioactive glass, bioactive glass ceramics, and calcium silicate [5-9]. Bioresorbable TCP material has been used as bone substitute due to its biocompatibility and osteoconductivity $[10,11]$.

In this report, we prepare new hybrid materials by combining stereocomplexation of PLLCL and PDLCL in the presence of TCP by supercritical fluid method. Here, we compare the non-grafted (pristine) TCP and PLLA-g-TCP as fillers in the stereocomplex matrix. The biocomposite from surface-modified TCP and stereocomplex matrix exhibits higher mechanical and thermal properties than the biocomposite from pristine TCP and stereocomplex matrix.

\section{Experimental section}

\subsection{Materials}

PDLCL $\left(M_{\mathrm{n}}=96,249 \mathrm{~g} / \mathrm{mol}, M_{\mathrm{w}}=239,118 \mathrm{~g} / \mathrm{mol}\right.$, $\mathrm{PDI}=2.484$, where PDI denotes the polydisperisty index $)$ and PLLCL $\left(M_{\mathrm{n}}=114,602 \mathrm{~g} / \mathrm{mol}, M_{\mathrm{w}}=\right.$ $252,362 \mathrm{~g} / \mathrm{mol}$, PDI $=2.202)$ with $10 \% \mathrm{~mol}$ of $\varepsilon$ caprolactone were synthesized by bulk polymerization in Biomaterial Research Center, Korea Institute of Science and Technology (KIST). TCP hydrate nano-powder (MFCD00015984) (Sigma-Aldrich, $<200 \mathrm{~nm}$ particle size, determined by the BrunauerEmmet-Tell/BET/method, USA) and phosphoric acid (MFCD00011340) (Sigma-Aldrich, $85 \mathrm{wt} \%$, USA), methylene chloride (9315-03) (JT Baker, HPLC grade, USA), and L-lactide (CAS No. 451142-6) (Medichem Chemical Co., Ltd., Korea, minimum purity $99.9 \%)$ and carbon dioxide $\left(\mathrm{CO}_{2}\right)$ (Shin Yang Oxygen Industry Co. Ltd., minimum purity $99.9 \%$, South Korea) were used as received.

\subsection{Grafting of lactide onto surface-modified TCP}

The surface-modified TCP was obtained by a method similar to one already described in the literature [12]. Briefly; TCP hydrate was vacuumed at $80^{\circ} \mathrm{C}$ for 24 hours before use. TCP powder was added to $5 \%$ aqueous phosphoric acid solutions $(1: 2 \mathrm{w} / \mathrm{w})$ and stirred at room temperature for 1 hour. The precipitate was removed from the solution by centrifugal separation. These powders were washed five times with distilled water to completely remove free residual $\mathrm{PO}_{4}{ }^{3-}$ ions, and dried at $60^{\circ} \mathrm{C}$ under vacuum before used. Evacuation was used to minimize residual water in order to reduce the formation of ungrafted PLLA and L-lactide hydrolisis [13, 14].

The dry surface-modified TCP powder was grafted by L-lactide through ring-opening polymerization using stannous octoate as catalyst. $8.4 \mathrm{~g}$ of L-lactide was mixed with $1 \mathrm{~g}$ of surface-modified TCP (molar ratio 1:10) and stannous octoate $(0.05 \mathrm{wt} \%)$ in a glass ampoule. The polymerization was carried out at $130^{\circ} \mathrm{C}$ for 36 and 96 hours. The product then washed by chloroform to separate the non-grafted PLLA, filtered, and dried at $50^{\circ} \mathrm{C}$ under vacuum. The polymerization resulted in PLLA-g-TCP.

\subsection{Preparation of stereocomplex-TCP biocomposites}

The formation of stereocomplex-TCP biocomposite was carried out by method using supercritical $\mathrm{CO}_{2}-$ methylene chloride similar to that described earlier [16]. A blend of PLLCL 10\% and PDLCL 10\% (weight ratio 1:1) was mixed with surface-modified TCP or pristine TCP and placed into a $40 \mathrm{~mL}$ stainless steel high pressure reactor equipped with a magnetic stirrer and electronic heating equipment. Methylene chloride was added to the reactor by syringe, and then the reactor was connected to the supercritical fluid apparatus. The reactor was filled with liquid $\mathrm{CO}_{2}$ at $30^{\circ} \mathrm{C}$ and 100 bar then gradually heated until reaching $65^{\circ} \mathrm{C}$ and 350 bar. The methylene chloride to $\mathrm{CO}_{2}$ ratio is $30: 70(\mathrm{wt} \%)$. The reaction process was allowed to proceed for the predetermined times $(5 \mathrm{~h})$ and the reactor was opened immediately after the reaction had finished.

\subsection{Characterization}

Surface modification of TCP was analyzed by IR spectrometer Nicolet iS10 (Thermo SCIENTIFIC). Polymer grafted onto the surface of TCP was confirmed by ToF-SIMS (time of flight secondary ion mass spectroscopy) analysis using ToF-SIMS 5 (ION-TOF). Thermal gravimetric analysis (TGA) was conducted on a Hi-Res TGA 2950 (TA Instrument, USA) under $\mathrm{N}_{2}$ flow at a heating rate of $10^{\circ} \mathrm{C} / \mathrm{min}$. Stereocomplex-composites formation 
was monitored by DSC (differential scanning calorimetry) using a DSC Q20 (TA Instrument, USA) under a $\mathrm{N}_{2}$ flow of $50 \mathrm{~mL} / \mathrm{min}$ at a heating rate $10^{\circ} \mathrm{C} / \mathrm{min}$. The crystalline structure of stereocomplex-composites with grafted or non-grafted TCP at varied content was analyzed by WAXD (wide angle X-ray diffraction), recorded on X-ray diffractometer Rigaku D/Max-2500 composed of $\mathrm{Cu} \mathrm{K} \mathrm{K}_{\alpha}(\lambda=1.54056 \AA \hat{,}, 30 \mathrm{kV}, 100 \mathrm{~mA})$ source, a quartz monochromator, and a goniometric plate. The mechanical properties of biocomposites were measured by an Instron apparatus with specimen size was $20 \times 5 \mathrm{~mm}$, and sample thickness about $10 \mu \mathrm{m}$. The distance between supports was $10 \mathrm{~mm}$ and the extension rate was $1 \mathrm{~mm} / \mathrm{min}$ for extension rate. Scanning electron microscopy (SEM) was used to evaluate the morphology of surface-modified TCP and TCP filler distribution in stereocomplex matrix using a FEI SEM INSPECT F50 (FEI).

\section{Results and discussion}

The attachment of inorganic materials to the polymer matrix is an important point in composite systems. Thus, surface-modification or polymer grafting is useful to improve the miscibility of those materials.

The surface activation followed by polymer grafting was applied to TCP materials. IR spectra were used to evaluate TCP, surface-activated TCP, PLLA, and PLLA-g-TCP as shown in Figure 1.

The surface-activated TCP was successfully synthesized from pristine TCP. The characteristic alterations in IR spectrum of surface-activated TCP in comparison with pristine TCP are observable. At the wavenumber below $1000 \mathrm{~cm}^{-1}$, the surface-activated TCP shows different spectrum at 643,933 ,

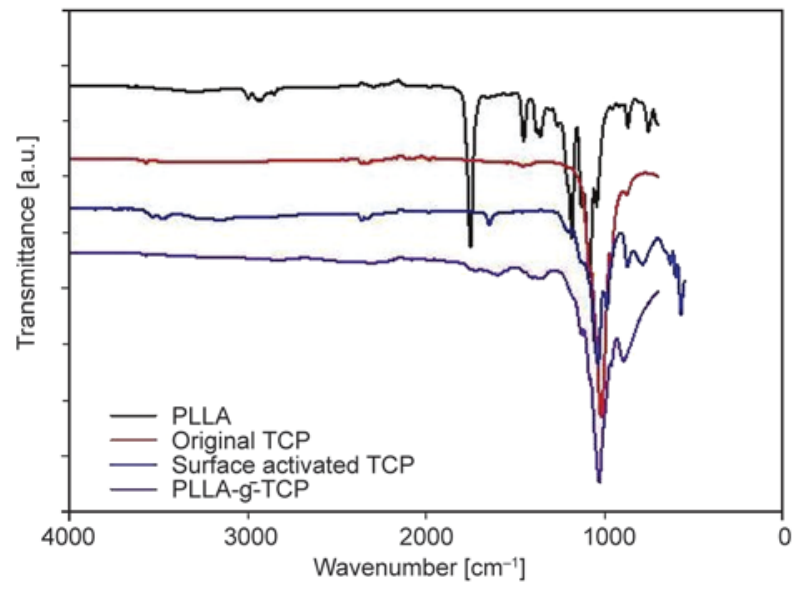
PLLA-g-TCP, and neat PLLA

and $997 \mathrm{~cm}^{-1}$ which correspond to $\mathrm{O}-\mathrm{P}-\mathrm{O}$ bending or $\mathrm{H}_{2} \mathrm{O}$ waging, $\mathrm{P}-\mathrm{O}$ stretching, and $\mathrm{H}_{2} \mathrm{O}$ rocking, respectively [12]. Low intensity of $\mathrm{O}-\mathrm{H}$ valence band in the region $3100-3600 \mathrm{~cm}^{-1}$ and band of crystal water of surface-activated TCP were clearly observed. The neat PLLA has the characteristic carbonyl band at $1750 \mathrm{~cm}^{-1}, \mathrm{C}-\mathrm{H}$ stretching band at 2920 and $3000 \mathrm{~cm}^{-1}$, and $\mathrm{C}-\mathrm{H}$ bending band at 1450 and $1360 \mathrm{~cm}^{-1}[13,14]$. The IR spectrum PLLA-g-TCP shows that PLLA was successfully grafted onto TCP surfaces. It can be seen as the dissapearance of hydroxyl band $\left(3100-3600 \mathrm{~cm}^{-1}\right)$ and the presence of small absorbance of carbonyl band, $\mathrm{C}-\mathrm{H}$ stretching, $\mathrm{C}-\mathrm{H}$ bending which come from PLLA characteristics.

The appearance of PLLA-g-TCP was shown in Figure 2. PLLA-g-TCP was successfully obtained by ring-opening polymerization by stannous octoate for 36 and $96 \mathrm{~h}$. The generated PLLA grafted TCPs from 36 and $96 \mathrm{~h}$ polymerization time were denoted as PLLA-g-TCP36 and PLLA-g-TCP96, respec-

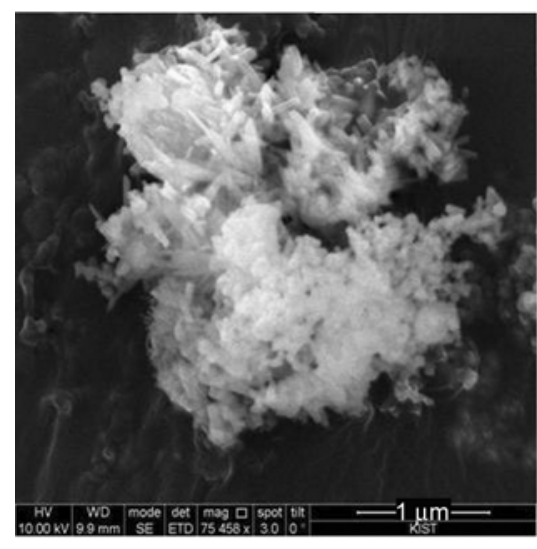

a)

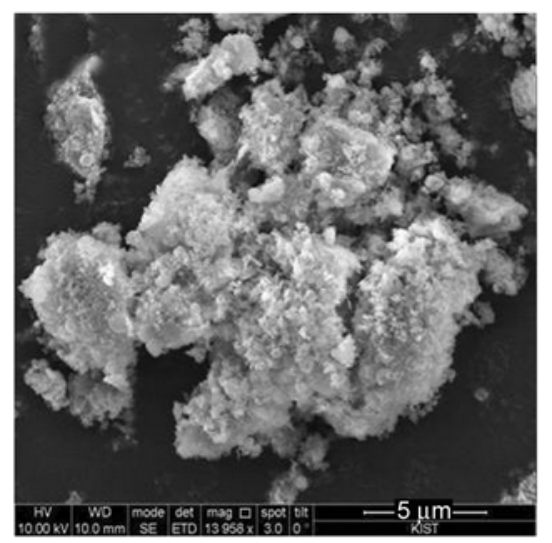

b)

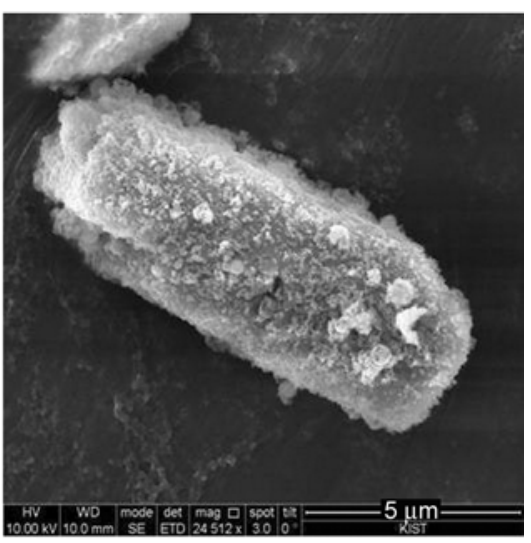

c)

Figure 2. SEM images: a) pristine TCP (scale bar $=1 \mu \mathrm{m})$; b) PLLA-g-TCP36 (scale bar $=5 \mu \mathrm{m})$; c) PLLA-g-TCP96 (scale bar $=5 \mu \mathrm{m}$ ) 
tively. The PLLA growth from the hydroxyl group on the activated TCP surfaces which acts as initiator. From the SEM picture, the PLLA fully attached to the activated TCP surfaces for PLLA-g-TCP36 and PLLA-g-TCP96. The attachment of PLLA chain onto TCP surface was also confirmed by ToFSIMS analysis. The negative ion spectra of TCP powder show the characteristic fragments of TCP (Figure 3). Characteristic peaks were observed at $m / z 31,47,36$, and 79 , corresponding to $\mathrm{P}^{-}, \mathrm{PO}^{-}$, $\mathrm{PO}_{2}{ }^{-}$, and $\mathrm{PO}_{3}{ }^{-}$respectively. It can be seen that the spectrum is dominated by the phosphor containing fragments. Other fragment containing $\mathrm{Ca}$ was found at $\mathrm{m} / z 119$ attributed to $\mathrm{CaPO}_{3}{ }^{-}$which is consistent with the typical spectrum of hydroxyapatite [15]. Figure 4 shows distinct series of ions characteristic of hydroxyapatite in this mass region. The low mass ions at $\mathrm{m} / \mathrm{z} 16$ and 17 correspond to $\mathrm{O}^{-}$and $\mathrm{OH}^{-}$, respectively. In the $\mathrm{m} / \mathrm{z}$ region between 100 and 120 , there are two fragments $\mathrm{C}_{3} \mathrm{H}_{4} \mathrm{O}_{2} \mathrm{P}^{-}(\mathrm{m} / \mathrm{z} 103)$ and $\mathrm{CaPO}-\mathrm{C}_{2} \mathrm{H}_{4}^{-}(\mathrm{m} / z$ 115) which and related as characteristic fragments of grafted TCP. A peak at $m / z 144$ is ascribed to $\mathrm{C}_{6} \mathrm{H}_{8} \mathrm{O}_{4}{ }^{-}$or single fragment of $\mathrm{L}$-lactide. Another fragment containing phosphor was found at $m / z 175\left(\mathrm{C}_{6} \mathrm{H}_{8} \mathrm{O}_{4} \mathrm{P}^{-}\right)$. The peak intensity of PLLA-g-TCP96 is higher than PLLA-gTCP36 which means the grafted PLLA content in the PLLA-g-TCP96 is higher than PLLA-g-TCP36. Further evaluation has been done by TGA to evaluate the amount of grafted PLLA as depicted in Figure 5. The grafting degree of PLLA onto activated TCP surface was quantitatively determined by the total mass loss of PLLA-g-TCP. The grafting degree depends on the reaction time. PLLA-g-TCP96 has a grafting degree $(\sim 12 \%)$ higher than PLLA-gTCP36 ( 8\%). This indicates that PLLA-g-TCP96 has more grafted PLLA than PLLA-g-TCP36.

Stereocomplex-TCP biocomposites were prepared by combining PDLCL and PLLCL in the presence of TCP in supercritical $\mathrm{CO}_{2}$-methylene chloride.
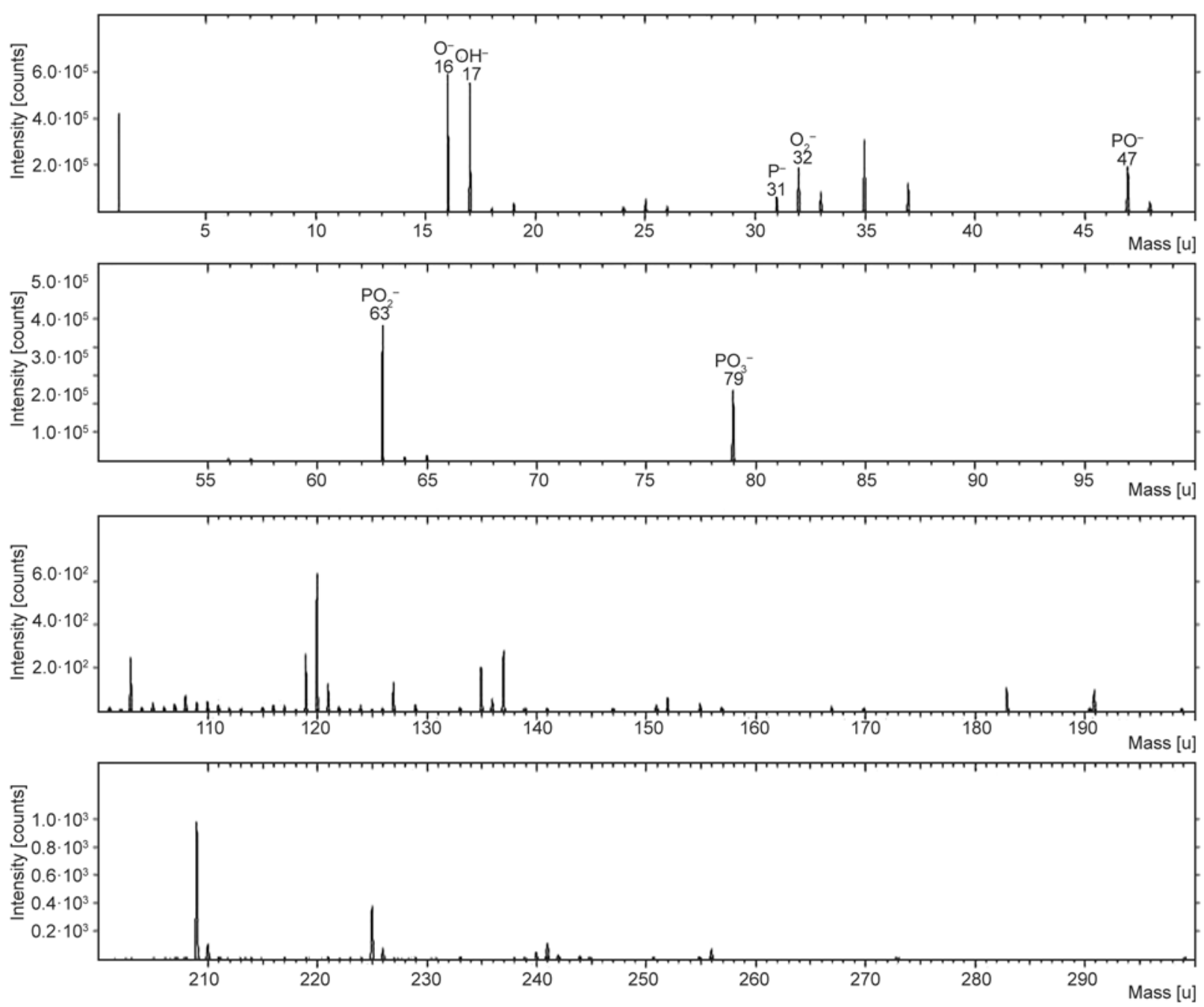

Figure 3. Negative ToF-SIMS spectrum of Pristine TCP 

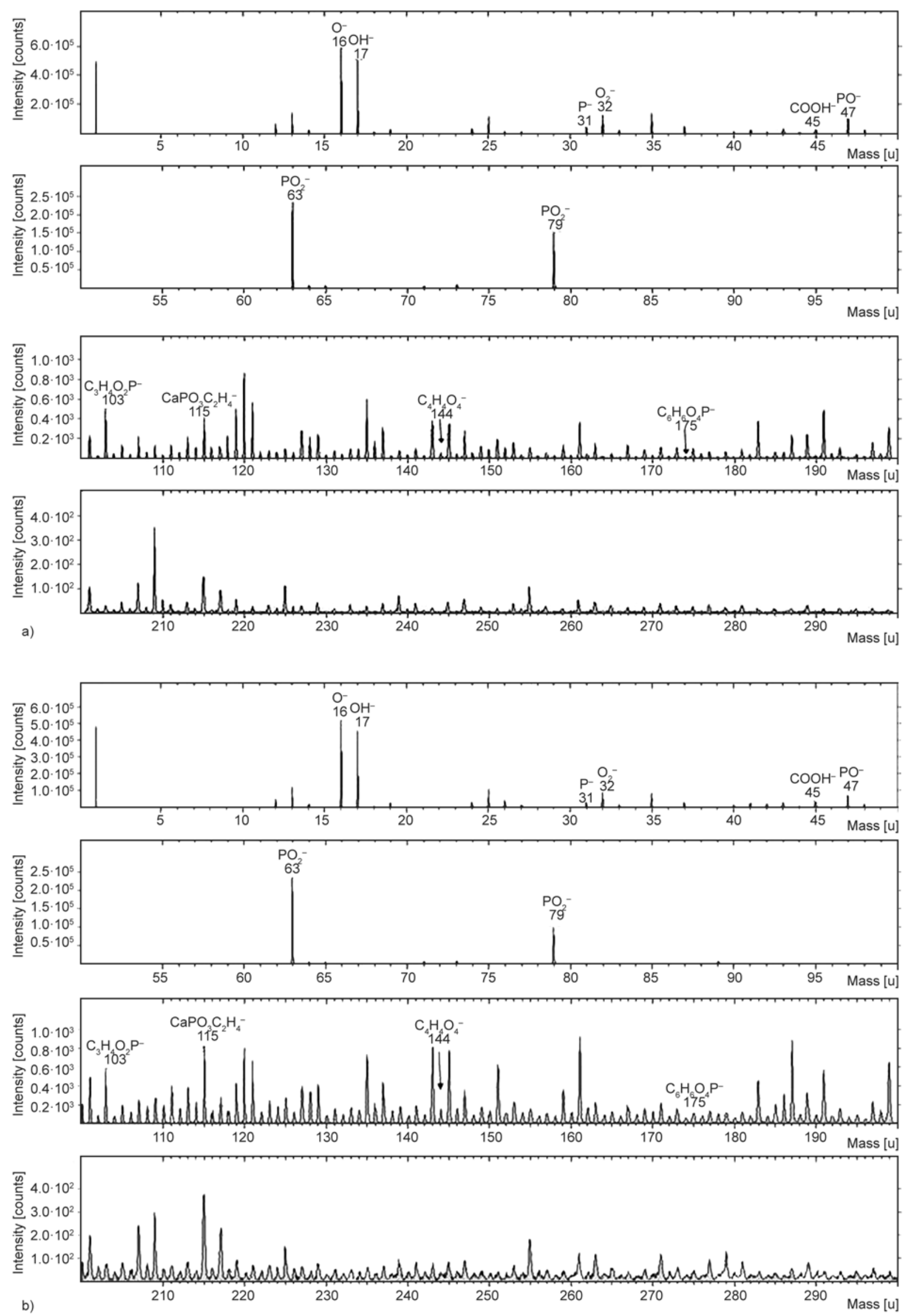

Figure 4. Negative ToF-SIMS spectra of PLLA-g-TCP36 (a) and PLLA-g-TCP96 (b) 


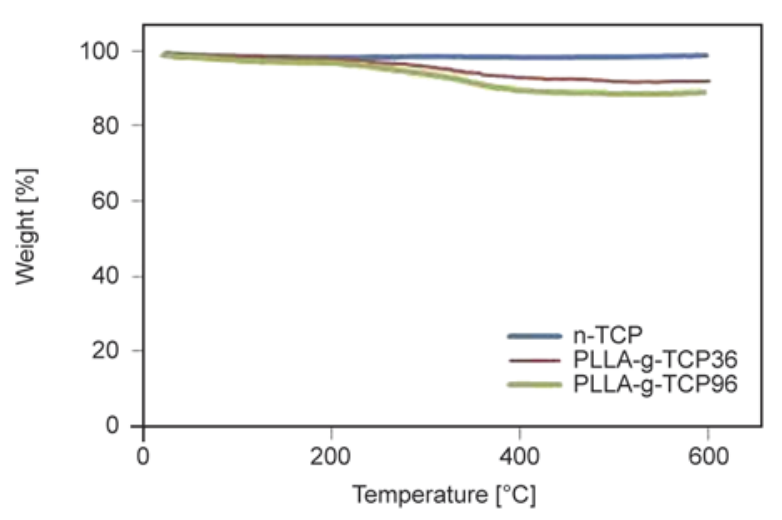

Figure 5. TGA thermograms of pristine TCP, PLLA-gTCP36, and PLLA-g-TCP96

Due to the limited solubility of PDLCL and PLLCL in supercritical $\mathrm{CO}_{2}$, methylene chloride was used as co-solvent to improve their solubility in supercritical $\mathrm{CO}_{2}$ [16]. PLLA-g-TCP96 and pristine TCP were used as inorganic material for these biocomposites due to the fact that they contain more grafted PLLA on the activated TCP surfaces compared to PLLA-g-TCP36. The presence of grafted-PLLA on activated-TCP surfaces is expected to improve the physical properties of generated-biocomposites.

The stereocomplex degree was evaluated by comparing $\Delta H_{\mathrm{m}}$ at the melting point $\left(T_{\mathrm{m}}\right)$ of the stereocomplex $\left(T_{\mathrm{m}}>200^{\circ} \mathrm{C}\right)$ and their homopolymers $\left(T_{\mathrm{m}}<180^{\circ} \mathrm{C}\right)$ from DSC anaysis. The neat s-PDLCL has a degree of stereocomplex of $\sim 88.36 \%$. It could not achieve $100 \%$ of stereocomplex during $5 \mathrm{~h}$ stereocomplexation processing time. As mentioned in the literature, the presence of $\varepsilon$-caprolactone fragments bothers the interaction between L- and D-lactide fragments to form stereocomplex [4]. In the biocomposite system, the presence of inorganic materials also affects the interaction with the polymer matrix [17].
The s-PDLCL containing PLLA-g-TCP96 (sPDLCL/g-TCP) shows higher stereocomplex degree than s-PDLCL containing pristine TCP (s-PDLCL/ TCP) as tabulated in Table 1. In the s-PDLCL/ TCPs, the presence of TCP reduces the degree of stereocomplex in the biocomposite. At 5\% TCP content, stereocomplex degree of s-PDLCL/TCP decreases drastically to about $\sim 44 \%$. The presence of $\varepsilon$-caprolactone fragments in the s-PDLCL results in decreasing stereocomplex degree compared to the streocomplex formed from polylactide homopolymer [4]. The presence of inorganic materials also caused a decreasing stereocomplex degree due to the interruption of the interaction between L- and D-lactide fragments to form stereocomplex [17]. The increasing TCP content decreases the stereocomplex degree proportionally. The increasing TCP content means the stereocomplex biocomposite system contains more particles which interfere with the stereocomplex formation. Thus, the presence of $\varepsilon-$ caprolactone fragments and TCP materials simultaneously caused the decreasing stereocomplex degree. The heat of melting $\left(\Delta H_{\mathrm{m}}\right)$ of s-PDLCL/g-TCP is higher than that of s-PDLCL/TCP. It is presumably caused by the nucleating effect of TCP particles in the polymer matrix. The PLLA-g-TCP exhibits better distribution in the polymer matrix than pristine TCP due to the presence of grafted-PLLA. The $\Delta H_{\mathrm{m}}$ of biocomposite is lower than that of s-PDLCL. The crystallinity depends on the arrangement of polymer chains [20]. The presence of TCP particle interferes with the arrangement of the polymer chain, which leads to decreasing $\Delta H_{\mathrm{m}}$.

s-PDLCL/g-TCP exhibits different behavior from sPDLCL/TCP. The presence of TCP materials in the PLLA-g-TCP96 form has different effect on the stereocomplex biocomposite system. Compared to

Table 1. The summary of DSC data for s-PDLCL, s-PDLCL/TCP, and s-PDLCL/g-TCP

\begin{tabular}{|l|c|c|c|c|c|c|c|}
\hline \multicolumn{1}{|c|}{ Materials } & $\begin{array}{c}\mathbf{T C P} \\
{[\mathbf{\%}]}\end{array}$ & $\begin{array}{c}\mathbf{T}_{\mathbf{g}} \\
{\left[{ }^{\circ} \mathbf{C}\right]}\end{array}$ & $\begin{array}{c}\mathbf{T}_{\mathbf{m}}{ }^{\mathbf{1}} \\
{\left[{ }^{\circ} \mathbf{C}\right]}\end{array}$ & $\begin{array}{c}\Delta \mathbf{H}_{\mathbf{m}}{ }^{\mathbf{1}} \\
{[\mathbf{J} / \mathbf{g}]}\end{array}$ & $\begin{array}{c}\mathbf{T}_{\mathbf{m}}{ }^{2} \\
{\left[{ }^{\circ} \mathbf{C}\right]}\end{array}$ & $\begin{array}{c}\Delta \mathbf{H}_{\mathbf{m}}{ }^{2} \\
{[\mathbf{J} / \mathbf{g}]}\end{array}$ & $\begin{array}{c}\text { Degree of } \\
\text { stereocomplex } \\
{[\mathbf{\%}]}\end{array}$ \\
\hline s-PDLCL & 0 & 44.86 & 149.70 & 5.358 & 205.53 & 40.67 & 88.36 \\
\hline s-PDLCL/TCP5 & 5 & 46.58 & 149.87 & 10.54 & 205.85 & 8.58 & 44.87 \\
\hline s-PDLCL/TCP10 & 10 & 51.27 & 155.09 & 15.23 & 201.61 & 8.53 & 35.90 \\
\hline s-PDLCL/TCP15 & 15 & 53.61 & 153.09 & 24.29 & 201.64 & 9.27 & 27.63 \\
\hline s-PDLCL/g-TCP5 & 5 & 45.86 & 150.27 & 1.519 & 202.57 & 16.73 & 91.68 \\
\hline s-PDLCL/g-TCP10 & 10 & 45.57 & 146.80 & 1.556 & 206.50 & 25.81 & 94.31 \\
\hline s-PDLCL/g-TCP15 & 15 & 46.37 & 145.41 & 1.759 & 206.59 & 25.09 & 93.34 \\
\hline
\end{tabular}

${ }^{1}$ copolymer

${ }^{2}$ stereocomplex 
s-PDLCL/TCP at 5\% TCP content, the s-PDLCL/gTCP composite has about $\sim 3 \%$ higher stereocomplex degree than s-PDLCL. The different behavior is caused by the presence of grafted-PLLA on the activated TCP surfaces. The grafted PLLA on activated TCP surfaces improves the miscibility of PLLA-g-TCP with the stereocomplex matrix. PLLAg-TCP96 exhibits better interfacial phase interaction in the stereocomplex biocomposite than pristine TCP [12]. The presence of grafted-PLLA on the activated TCP surfaces also supports the formation of stereocomplex with D-lactide fragment which results in increasing stereocomplex degree $(\sim 3 \%)$. The increasing TCP contents of s-PDLCL/g-TCP increase the stereocomplex degree up to a certain level, and then slightly decrease. In the s-PDLCL/gTCP biocomposite system, it assumed that graftedPLLA and TCP materials have opposite effects on stereocomplexation. The grafted-PLLA supports the miscibility and stereocomplex formation. Contrary, TCP materials tend to disrupt the L- and Dlactide fragments in the system. At the certain content (up to 10\%) of PLLA-g-TCP96, the effect of grafted-PLLA seems to be superior to that of TCP. But, at higher TCP content (15\%), the TCP effect starts to overcome the grafted-PLLA effect. That counter-effect causes the increasing of stereocomplex degree up to $10 \%$ of TCP content, then the decrease at higher TCP content (15\%).

WAXD analysis was performed for the further investigation for the stereocomplex structure in the presence of calcium phosphate filler. The neat copolymers (PLLCL and PDLCL) show diffraction peaks

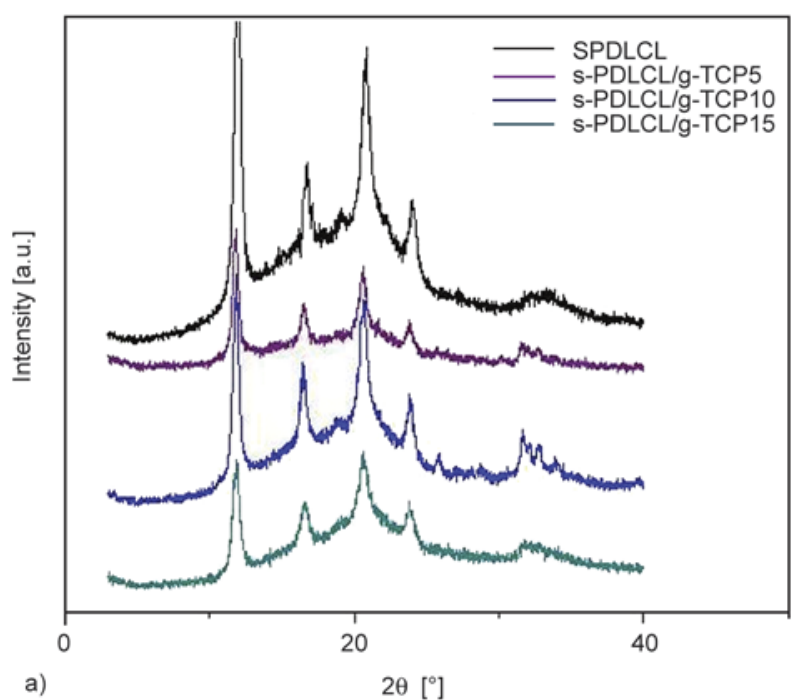

at $2 \theta=16.56,18.74$, and $21.74^{\circ}$ (figure not shown) corresponding to the $\alpha$-form of the copolymer. The stereocomplex biocomposites and s-PDLCL (control sample) exhibit appearance of new peaks at $2 \theta=$ $11.96,20.82$, and $23.88^{\circ}$ which correspond to stereocomplex crystallites $[14,15]$. The presence of PLLA-g-TCP96 increases stereocomplex degree of the s-PDLCL/g-TCP biocomposites up to a certain PLLA-g-TCP content (Figure 6a). It was shown by the increasing relative intensity of peaks characteristic of the stereocomplex as compared to that in the copolymer. The grafted-PLLA fragments on the activated TCP surface can interact with D-lactide fragments from copolymer PDLCL and form stereocomplex crystallites. PLLA-g-TCP96 can act as a heterogeneous nucleating agent and decreases the crystalization activation energy up to certain content. Above the optimum content, PLLA-g-TCP96 can act as blocking site which obstructs the formation of stereocomplex crystallites. In the other hand, the WAXD results for s-PDLCL/TCP (Figure 6b) show the decreasing relative intensity of stereocomplex relative to copolymer peaks which means decreasing stereocomplex degree by increasing TCP contents.

The distribution of inorganic TCP in the stereocomplex matrix is illustrated by SEM images in Figure 7. For s-PDLCL/TCP biocomposites (Figure $7 \mathrm{a}-7 \mathrm{c}$ ), pristine TCP has limited dispersibility in the stereocomplex matrix. There are some TCP particle agglomerates even at 5\% TCP content which caused by the limited interaction between inorganic TCP with polymer matrix. The grafted-PLLAs sup-

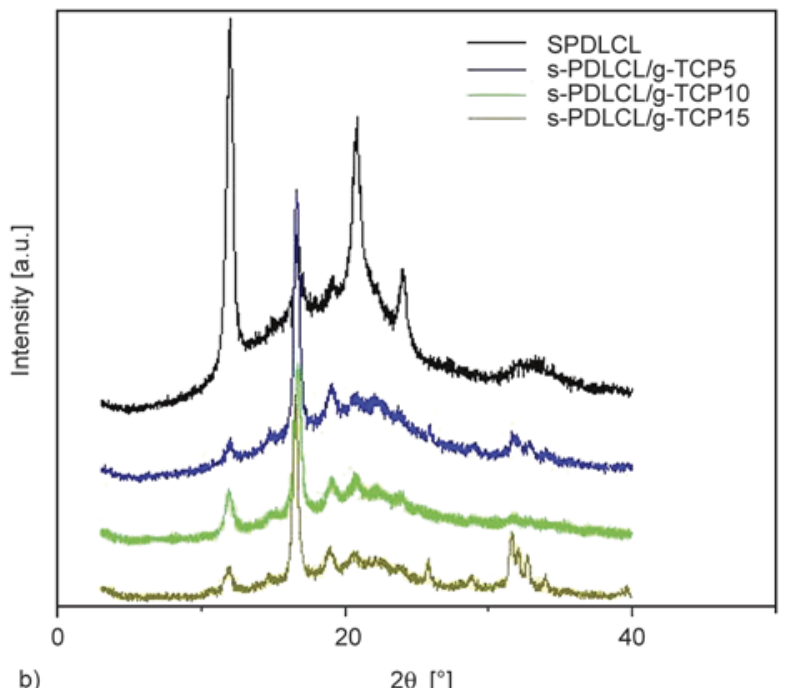

b)

Figure 6. WAXD patterns of biocomposites: a) s-PDLCL/g-TCP; b) s-PDLCL/TCP 


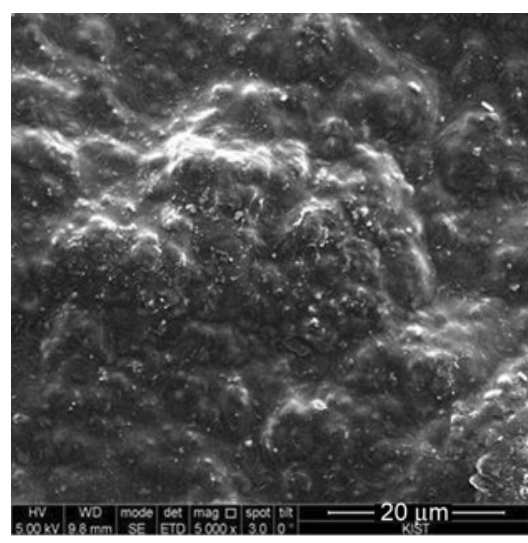

a)

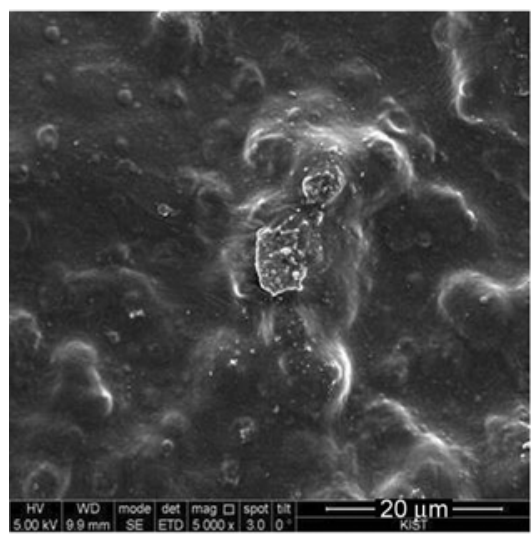

d)

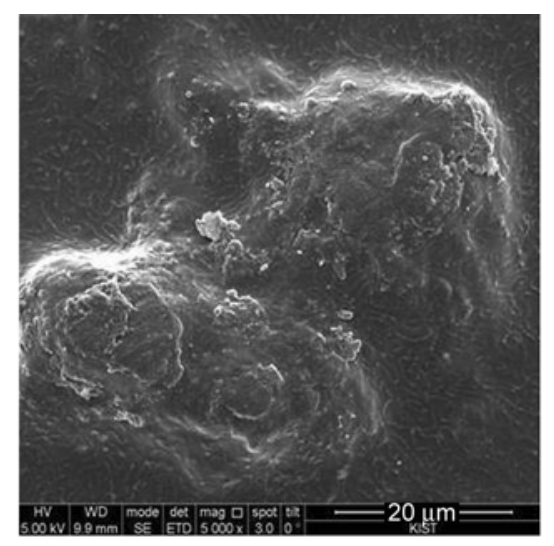

b)

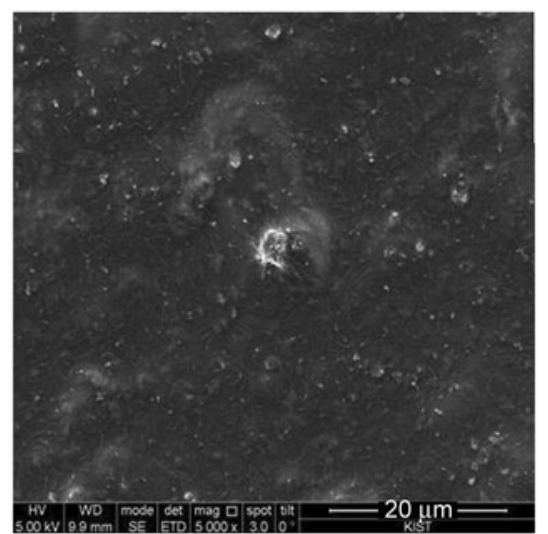

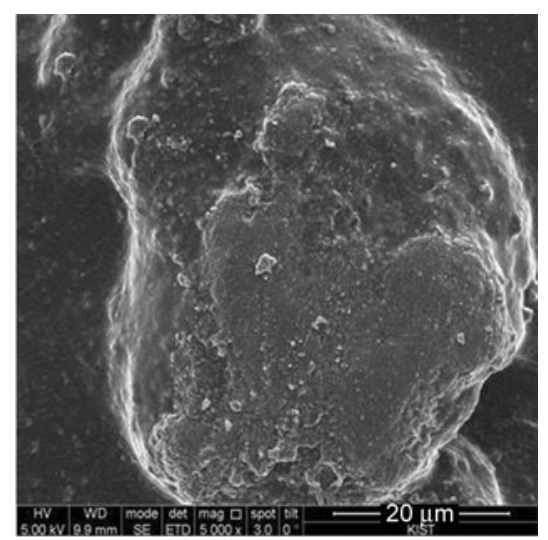

c)

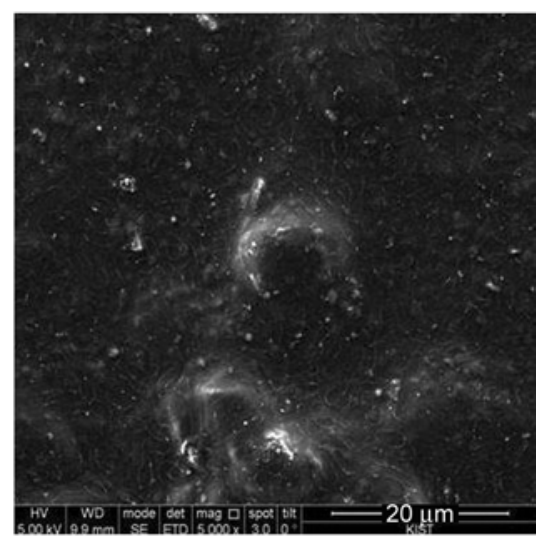

f)

Figure 7. SEM images of s-PDLCL/TCP: a) 5\%; b) 10\%; c) $15 \%$ of TCP particles and s-PDLCL/g-TCP biocomposites: d) $5 \%$; e) $10 \%$; f) $15 \%$ of PLLA-g-TCP (scale bar $=20 \mu \mathrm{m}$ )

port the dispersion of TCP in the stereocomplex matrix as shown in Figure 7d-7f. The grafted-PLLA has strong interaction with L-lactide fragments from PLLCL and also D-lactide fragments from PDLCL by forming stereocomplex. Those interactions result in homogeneous dispersion of PLLA-gTCP in the stereocomplex biocomposite matrix.

The addition of inorganic materials to the polymeric matrix is practiced to improve its mechanical properties. The Young's modulus of s-PDLCL and biocomposites from s-PDLCL and inorganic TCP was depicted in Figure 8. The s-PDLCL/g-TCPs show higher Young's modulus compare to the sPDLCL/TCP and neat s-PDLCL. The s-PDLCL/ TCP shows slight improvement in Young's modulus even though the stereocomplex degree was decreased with increasing TCP content. It also has limitation in making homogeneous dispersion in stereocomplex matrix. The small improvement in Young's modulus was presumably supported by the stiffening effect of TCP materials [18]. Otherwise, sPDLCL/g-TCPs show better improvement in Young's modulus. The Young's modulus was increased by increasing TCP content up to certain

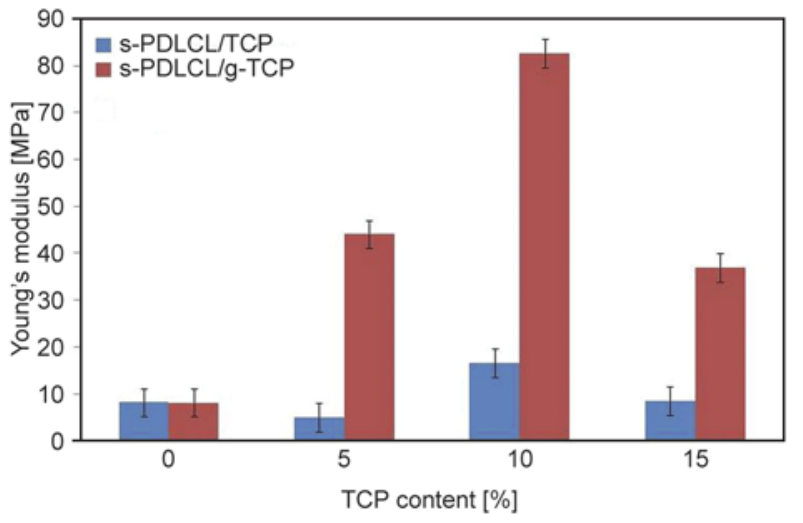

Figure 8. The mechanical property (Young's modulus) of sPDLCL and its biocomposites (s-PDLCL/TCP and s-PDLCL/g-TCP)

content $(10 \%)$, then decreased at higher content $(15 \%)$. The maximum Young's modulus was $\sim 80 \mathrm{MPa}$ at $10 \%$ of PLLA-g-TCP96 content. This mechanical improvement is in accordance with the result from DSC analysis and SEM images. The presence of grafted-PLLA from PLLA-g-TCP96 in the s-PDLCL/g-TCP results in a homogeneous dispersion of TCP in the stereocomplex matrix and also increases the stereocomplex degree. The decreasing Young's modulus at s-PDLCL/g-TCP15 
was caused by the decrease of the stereocomplex degree and by the presence of TCP particle in high concentration $(15 \%)$. There is a slight decrease in the stereocomplex degree of s-PDLCL/g-TCP. On the other hand, higher solid filler (PLLA-g-TCP) in the composite tends to form aggregate which decreases the interfacial filler-polymer matrix adhesion [19].

\section{Conclusions}

PLLA-g-TCP was successfully produced by ringopening polymerization of L-lactide in the presence of surface-activated TCP. The attachment of PLLA was confirmed by IR spectroscopy, ToF-SIMS, and SEM images. Stereocomplex biocomposites were obtained by combining PDLCL and PLLCL in the presence of pristine TCP or PLLA-g-TCP96 through supercritical $\mathrm{CO}_{2}$-methylene chloride system. The s-PDLCL/TCPs show decreasing in stereocomplex degree and inhomogeneous dispersion of TCP in stereocomplex matrix which results in a slight improvement of the mechanical properties. Contrary, the presence of PLLA-g-TCP96 in the s$\mathrm{PDLCL} / \mathrm{g}$-TCP increases the stereocomplex degree up to certain content and also supports the homogeneous dispersion of TCP materials in stereocomplex matrix. These interactions support the improvement of mechanical properties of s-PDLCL/g-TCP. The optimum content of PLLA-g-TCP96 in s-PDLCL/ $\mathrm{g}$-TCP biocomposite is $10 \%$. The combination of stereocomplex matrix and TCP materials provide a promising route to use such materials for internal fracture fixation devices.

\section{Acknowledgements}

This study was supported by the National Research Foundation of Korea Grant funded by the Korea Government (MEST), NRF-2010-C1AAA001-0028939.

\section{References}

[1] Törmälä P., Pohjonen T., Rokkanen P.: Bioabsorbable polymers: Materials technology and surgical applications. Proceedings of the Institution of Mechanical Engineers Part H: Journal of Engineering in Medicine, 212, 101-111 (1998).

DOI: $10.1243 / 0954411981533872$
[2] De Jong S. J., van Dijk-Wolthuis W. N. E., Kettenesvan den Bosch J. J., Schuyl P. J. W., Hennink W. E.: Monodisperse enantiomeric lactic acid oligomers: Preparation, characterization, and stereocomplex formation. Macromolecules, 31, 6397-6402 (1998). DOI: $10.1021 / \mathrm{ma980553 \textrm {i }}$

[3] Tsuji H.: Poly(lactide) stereocomplexes: Formation, structure, properties, degradation, and applications. Macromolecular Bioscience, 5, 569-597 (2005). DOI: 10.1002/mabi.200500062

[4] Purnama P., Jung Y., Kim S. H.: Stereocomplexation of poly(L-lactide) and random copolymer poly(D-lactide-co-e-caprolactone) to enhance melt stability. Macromolecules, 45, 4012-4014 (2012).

DOI: $10.1021 / \mathrm{ma} 202814 \mathrm{c}$

[5] Qiu X., Chen L., Hu J., Sun J., Hong Z., Liu A., Chen X., Jing X.: Surface-modified hydroxyapatite linked by L-lactic acid oligomer in the absence of catalyst. Journal of Polymer Science Part A: Polymer Chemistry, 43, 5177-5185 (2005).

DOI: $10.1002 /$ pola.21006

[6] Miyai T., Ito A., Tamazawa G., Matsuno T., Sogo Y., Nakamura C., Yamazaki A., Satoh T.: Antibioticloaded poly- $\varepsilon$-caprolactone and porous $\beta$-tricalcium phosphate composite for treating osteomyelitis. Biomaterials, 29, 350-358 (2008).

DOI: 10.1016/j.biomaterials.2007.09.040

[7] Li X., Shi J., Dong X., Zhang L., Zeng H.: A mesoporous bioactive glass/polycaprolactone composite scaffold and its bioactivity behavior. Journal of Biomedical Materials Research Part A, 84, 84-91 (2008). DOI: $10.1002 / \mathrm{jbm} . \mathrm{a} .31371$

[8] Oréfice R., Clark A., West J., Brennan A., Hench L.: Processing, properties, and in vitro bioactivity of polysulfone-bioactive glass composites. Journal of Biomedical Materials Research Part A, 80, 565-580 (2007).

DOI: $10.1002 / \mathrm{jbm} . \mathrm{a} .30948$

[9] Huan Z., Chang J.: Self-setting properties and in vitro bioactivity of calcium sulfate hemihydrate-tricalcium silicate composite bone cements. Acta Biomaterialia, 3, 952-960 (2007).

DOI: $10.1016 /$ j.actbio.2007.05.003

[10] Low K. L., Tan S. H., Zein S. H. S., Roether J. A., Mouriño V., Boccaccini A. R.: Calcium phosphatebased composites as injectable bone substitute materials. Journal of Biomedical Materials Research Part B: Applied Biomaterials, 94, 273-286 (2010). DOI: $10.1002 / \mathrm{jbm}$. b. 31619

[11] Hak D. J.: The use of osteoconductive bone graft substitutes in orthopaedic trauma. Journal of the American Academy of Orthopaedic Surgeons, 15, 525-536 (2007).

[12] Choudhery R. A., Vance C. J.: The characterisation and properties of modern conversion coatings for use with paint films. in 'Advances in corrosion protection by organic coatings. Cambridge, England' 64 (1989). 
[13] Kunze C., Freiter T., Helwig E., Sandner B., Reif D., Wutzler A., Radusch H-J.: Surface modification of tricalcium phosphate for improvement of the interfacial compatibility with biodegradable polymers. Biomaterials, 24, 967-974 (2003).

DOI: $10.1016 / \mathrm{s} 0142-9612(02) 00433-7$

[14] Hong Z., Qiu X., Sun J., Deng M., Chen X., Jing X.: Grafting polymerization of L-lactide on the surface of hydroxyapatite nano-crystals. Polymer, 45, 66996706 (2004).

DOI: $10.1016 /$ j.polymer.2004.07.036

[15] Eriksson C., Bömer K., Nygren H., Ohlson K., Bexell U., Billerdahl N., Johansson M.: Studies by imaging TOF-SIMS of bone mineralization on porous titanium implants after 1 week in bone. Applied Surface Science, 252, 6757-6760 (2006).

DOI: 10.1016/j.apsusc.2006.02.194

[16] Purnama P., Kim S. H.: Stereocomplex formation of high-molecular-weight polylactide using supercritical fluid. Macromolecules, 43, 1137-1142 (2010). DOI: $10.1021 / \mathrm{ma} 902536 \mathrm{p}$
[17] Purnama P., Lim S. H., Jung Y., Kim S. H.: Stereocomplex-nanocomposite formation of polylactide/fluorinated-clay with superior thermal property using supercritical fluid. Macromolecular Research, 60, 545-548 (2012).

DOI: 10.1007/s13233-012-0092-4

[18] Wilberforce S. I. J., Finlayson C. E., Best S. M., Cameron R. E.: A comparative study of the thermal and dynamic mechanical behaviour of quenched and annealed bioresorbable poly-L-lactide/ $\alpha$-tricalcium phosphate nanocomposites. Acta Biomaterialia, 7, 2176-2184 (2011).

DOI: $10.1016 /$ j.actbio.2011.02.006

[19] López Manchado M. A., Valentini L., Biagiotti J., Kenny J. M.: Thermal and mechanical properties of single-walled carbon nanotubes-polypropylene composites prepared by melt processing. Carbon, 43, 1499-1505 (2005).

DOI: $10.1016 / \mathrm{j}$.carbon.2005.01.031

[20] Odian G.: Principles of polymerization. Wiley, New York (1991). 